\title{
Performance enhancement of sensor network architecture for monitoring underwater oil pipeline
}

\author{
Waseem M. Jassim, Ammar E. Abdelkareem
}

Department of Networks Engineering, Al-Nahrain University, Iraq

\begin{tabular}{l} 
Article Info \\
\hline Article history: \\
Received Dec 24, 2019 \\
Revised Jul 13, 2020 \\
Accepted Sep 24, 2020 \\
\hline Keywords: \\
Improved smart redirect or \\
jump algorithm \\
Oil pipeline monitoring \\
Underwater wireless sensor \\
network
\end{tabular}

\begin{abstract}
In this paper, a deployment mechanism is designed to distribute heterogeneous nodes to optimally cover the pipeline where the mechanism helps locate each node on the wall of the oil pipeline where the number of nodes can be increased depending on this mechanism. The six-layer network hierarchy includes basic sensor nodes (BSN), aggregation relay node (ARN) that added to the network hierarchy, data relay nodes (DRN), data dissemination node (DDN), base station (sinks), and network control center (NCC). This network relies on the improved smart redirect or jump algorithm (SRJ) by sending packets depend on the active relay nodes in both directions that are within the transmission range of the ARNs instead of relying on the number of hops adopted by the SRJ algorithm to reduce the network delay, the energy consumed in relay nodes, and the number of times the DRNs increased transmission range. The OMNeT++ and MATLAB programs were used to implement the simulation scenario. The results showed superiority in terms of the average overhead communication, energy consumption, and end to the end delay with network delay in some cases rely on the number of active relay nodes.
\end{abstract}

This is an open access article under the $\underline{C C B Y-S A}$ license.

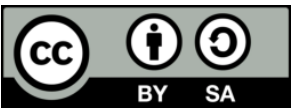

\section{Corresponding Author:}

Waseem M. Jassim

Department of Networks Engineering

Al-Nahrain University

Al jadria Road, Baghdad, Iraq

Email: wassim.hwm@gmail.com

\section{INTRODUCTION}

The monitoring of oil pipelines is very important according to the importance of oil transported through these pipelines, which represents a huge economic need for producing countries and consuming countries. The use of copper wires in the connections of the underwater sensor networks with radio signals or acoustic signals may lead to increased cost, the difficulty of maintenance and installation [1]. UWSN is used to provide a system with low cost and no need for a complex system in underwater for leakage, pollution, and exploration [2]. The Marines environment is difficult, thus the communication by an acoustic signal is expensive [3]. Many factors affect the acoustic signals including shallow water, salinity, the depth of diffusion of sensors, multipath, doppler, and noise from several sources, all these factors increase bits error, propagation delay and increased attenuation [4]. Linear networks are used to monitor the oil pipeline which based on limited routing protocols because of their linearity [5].

The smart redirect or jump algorithm (SRJ) is designed by combining the work of the jumping always algorithm and the redirect always algorithm. In the SRJ algorithm, the energy consumed in each direction depends on the number of hops in as each node has to decide the direction of the packets depending on the energy consumption in each direction [6]. The main problems in the SRJ algorithm are energy 
consumed when data relay node starts the process of relay data to the neighbor nodes, increasing in the number of jumps in the relay nodes as the case of no active neighbor relay node available in default directions, and in addition, sensor nodes connected with relay nodes may be lost. The aim of this work is to improve the hierarchical network model of the linear structure sensor network by adding new nodes to the hierarchy of the child/parent relationship. Adding these nodes, need to new deployment scheme for all the nodes in the network to cover the oil pipeline perfectly. The deployment mechanism is designed where every node in the network is assigned a location, and improve the (SRJ) algorithm so that these added nodes receive data and determine the direction of their transmission to the relay nodes and depend on the availability of active relay nodes, not on the number of jumps in each path as in the SRJ algorithm, and, accordingly, increase network reliability.

\section{RELATED WORK}

Mohamed and Jawhar [7], presents and analysis of the combination of wiring with acoustic and radio signals to provide a reliable communication, to compare network connectivity, meet harsh underwater environment challenges, power supply continuity for network, and the security of physical network. The jumping or redirect protocol (SRJ) is implemented and compared with the previous algorithms to increase the reliability of network. Ted Tsung-Te Lai provided a mobile wireless sensor network system to deploy independent sensors to monitor pipelines. Triops networks by automatically firing sensor nodes from a central repository located at the pipeline source. The sensor diffusion algorithm determines the location of the node within the tube. TriopusNet replaces the node from the central repository if its battery is low or experiences a fault.

Zahid [8] proposed a dynamic node deployment algorithm where a site is assigned to each node in a fast and efficient manner without the need for a localization scheme. This method provides an option for dealing with heterogeneous types of nodes and distributing the structure and mechanism by which new nodes can be easily added. Mohsin Murad provided analyzed study of underwater wireless sensor networks applications and the deployment of UAWSN networks to monitor and control all underwater areas, including monitoring of pipelines through linear sensor networks in all methods presented for data collection and sent to the surface sink, therefore, to the data collection center. Zahoor Ahmed and Kamalrulnizam Abu Bakar [9] presented an enhanced algorithm for the deployment mechanism of a linear pipeline using an underwater wireless sensor network (EULWSND). This study is to improve the strategies for deployment linear underwater sensor data, as it was compared with other strategies taking into consideration the heterogeneity of the nodes and the linearity of the underwater pipelines.

\section{RELAYING ALGORITHM FOR LINEAR WIRELESS SENSOR NETWORK}

In this section, some routing algorithms that are used to overcome failures in relay nodes are reviewed to know how each algorithm works in relay nodes and the process of routing packets to the sinks [10].

\subsection{Jump algorithm (JA)}

To overcome the disconnection with neighboring relay nodes, relay nodes increase the transmission capacity and double the transmission range to reach the active relay nodes that follow the neighboring nodes. If multiple consecutive links are missing, in this case, the migration nodes can use a maximum multiplex of the transmission range to bypass disabled nodes and access the active nodes. The maximum hop count depends on a coefficient based on the network variable that represents the number of nodes that are disabled. If no active module is reached, the message is dropped.

\subsection{Redirect algorithm (RA)}

This routing algorithm differs because it relies on message redirect in relay nodes instead of increasing the transmission range. The network relies on a hierarchical structure of two parents that can route the message to the opposite end if there is no active neighboring node in the default direction. If there are no neighboring nodes in both directions, the message will drop.

\subsection{Smart redirect or jump algorithm (SRJ)}

This algorithm is designed by combining the work of the jumping algorithm and the redirect algorithm to overcome the failure situation in relay nodes. In this algorithm, each node save information of the neighboring nodes. The routing decision is made by relay nodes depending on the energy consumed in each direction. The energy consumed in each direction depends on the number of jumps in each direction as 
each node has to decide the direction of the packets depending on the energy consumption for each direction to reach the sink node. The message is sent by the sensors to the relay node, which in turn calculates the number of hops for each direction where the relay node chooses the least number of hops. If the node fails to reach the neighboring node in the default direction, the relay node redirects the message to the opposite direction to reach the secondary parent. Figure 1 shows the work of the SRJ algorithm in a linear wireless sensor network (LWSN).

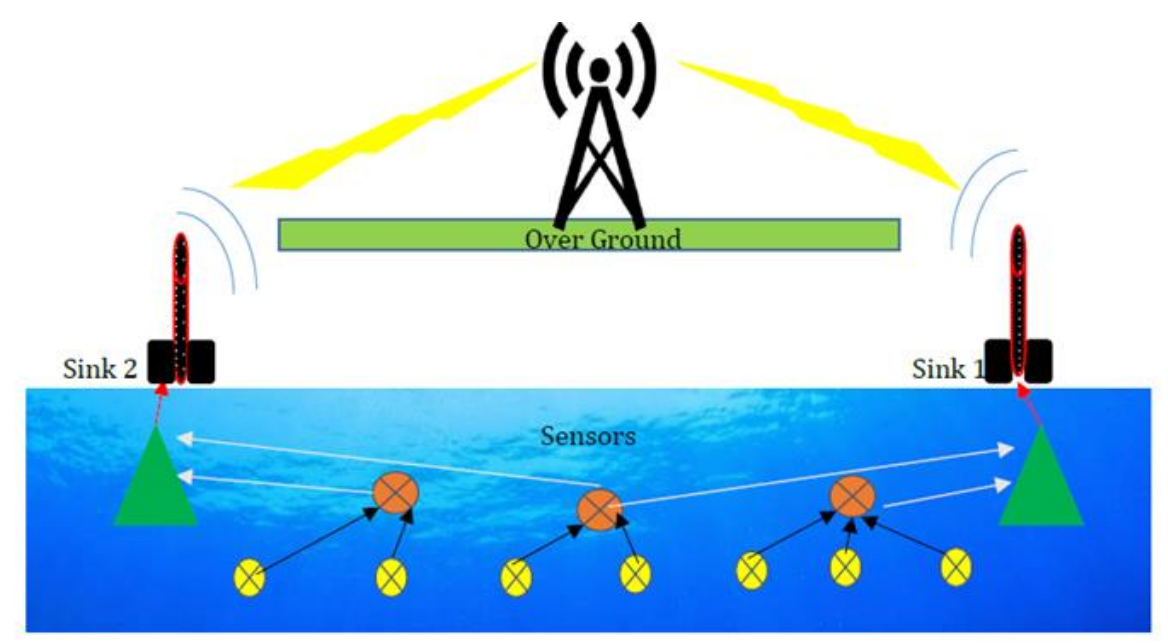

Figure 1. LWSN based SRJ algorithm [10]

\subsubsection{Hierarchical sensor network design}

The SRJ algorithm that works depending on the relationship between child/parents [11, 12]. Therefore, the hierarchical structure of the network consists of three different types of heterogeneous nodes that are responsible for the process of sensing, relaying, and dissemination. These nodes are connected to the sink up to the network control center (NCC). Figure 2 shows the child/parent's hierarchical network model.

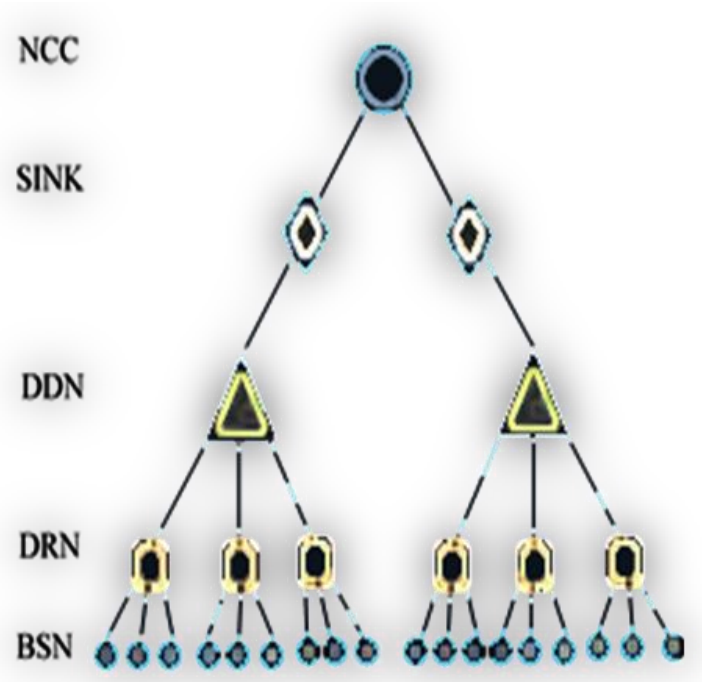

Figure 2. Child/parent's hierarchical network model [11]

Basic sensing nodes (BSNs): These nodes are used in several areas where they are deployed along the wall of the oil pipeline to monitor for leakage and corrosion to the oil pipeline. These nodes are sensitive and send information to the ARN node. 
Data relay nodes (DRNs): The function of this node is to receive data from sensor nodes and as these nodes decide the direction of sending packets, depending on the amount of energy consumed in both directions. These nodes relaying packets to the neighboring relay node to reach one of the data dissemination nodes (DDN) on the two sides of the oil pipeline. Data dissemination nodes (DDNs): These nodes are used to collect data from the relay data nodes and send them to the base stations (sink) in order to send data to the network control center (NCC).

\title{
3.3.2. Messages relaying strategy
}

For the SRJ strategy, the relay nodes relayed data based on energy-consumed in both directions thus, each relay node has a primary parent (DDN1) in default direction and secondary parent (DDN2) in opposite direction $[13,14]$. Data relay node DRN receives the packet from BSNs where DRN relaying data to neighbors. If the neighbor node is not active in the default direction, the jump algorithm is worked to connect the other DRNs. If there are no available nodes, in this case, the DRN uses redirect strategy. Each DRN has information about the neighboring nodes. The relaying of packets based on the energy-consumption (Ex) in default and opposite directions. For $\mathrm{Ex}^{1 \mathrm{p}}<\mathrm{Ex}^{2 \mathrm{p}}$, the direction of the packet is towards (DDN1), else, the packet will be towards (DDN2). Figure 3 shows the direction of the packet where the energy-consuming based on the number of hops in each direction. Therefore, each node should calculate the energy consumed in both directions to choose the lowest energy-consuming path, thus, increasing the delivery of packets to the parent.

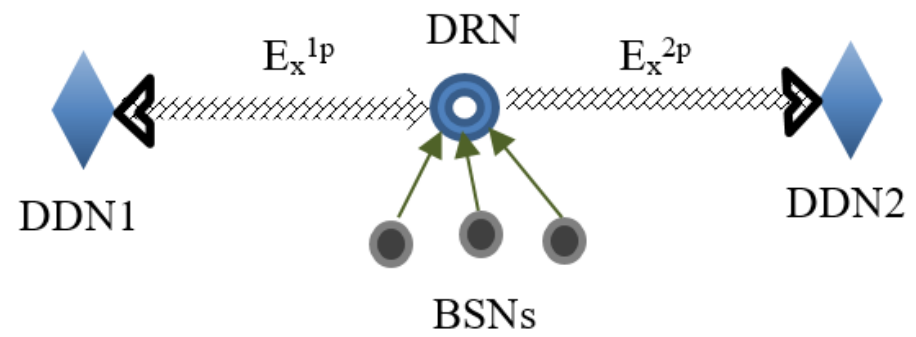

Figure 3. Packets direction based on energy-consumed in both directions [11]

\section{PROPOSED METHODOLOGY}

Underwater networks depend on how nodes are deployed to ensure coverage and communication between nodes. The deployment scheme of the nodes for a 1,000 meter oil pipeline includes the deployment of sensors from the point 25 meters, and the sensors are deployed every 50 meters. For the DRNs, the deployment scheme starts from the point 50 meters and are deployed every 100 meters. ARNs are deployed starting from the point 100 meters and deployed every 200 meters. As for the DDNs, the deployment scheme is at point 0 meters and 1,000 meters. Figure 4 shows the deployment of heterogeneous nodes around the wall of oil pipeline by determining the transmission ranges of these nodes.

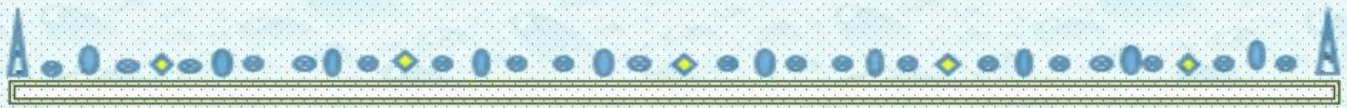

\author{
Basic Sensor Node \\ Aggregation Relay Node \\ Data Relay Node \\ Data Dissemination Node
}

Figure 4. Deployment scheme of the nodes around the oil pipeline

In this network, the sensors are connected to the aggregation relay node (ARN) which added to a hierarchical network model. Figure 5 represents the hierarchical of a new network model. This node collects and sends data to the DRNs depending on the active nodes instead of old strategy which depends on the 
number of jumps per directions. Network data does not pass through ARN, as in the relay nodes, but only data from connected sensors. The routing of data received from the sensors by the ARN depends on the number of data relay nodes connected to this node.

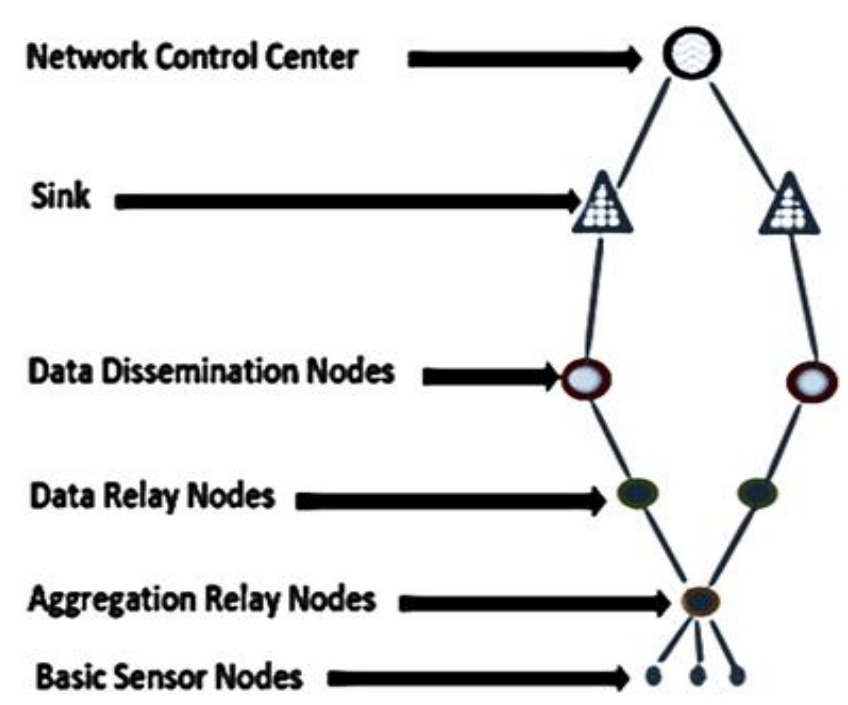

Figure 5. The hierarchical of proposed network model

In the proposed hierarchical network model, sensor nodes remain connected to the network if the relay nodes fail because they are linked with the (ARN) node that is connected to other DRNs. The overhead communication process between the nodes and the sensors is less if the sensors are linked to the ARN nodes. Figure 6 shows the improved SRJ algorithm (ISRJ) worked in the (ARN) node. This algorithm relies on the status of the DRNs on both directions. In the ISRJ algorithm, the packets are sent and the process of change direction is done before the jumping mode, therefore, reducing the energy consumed in ARNs.

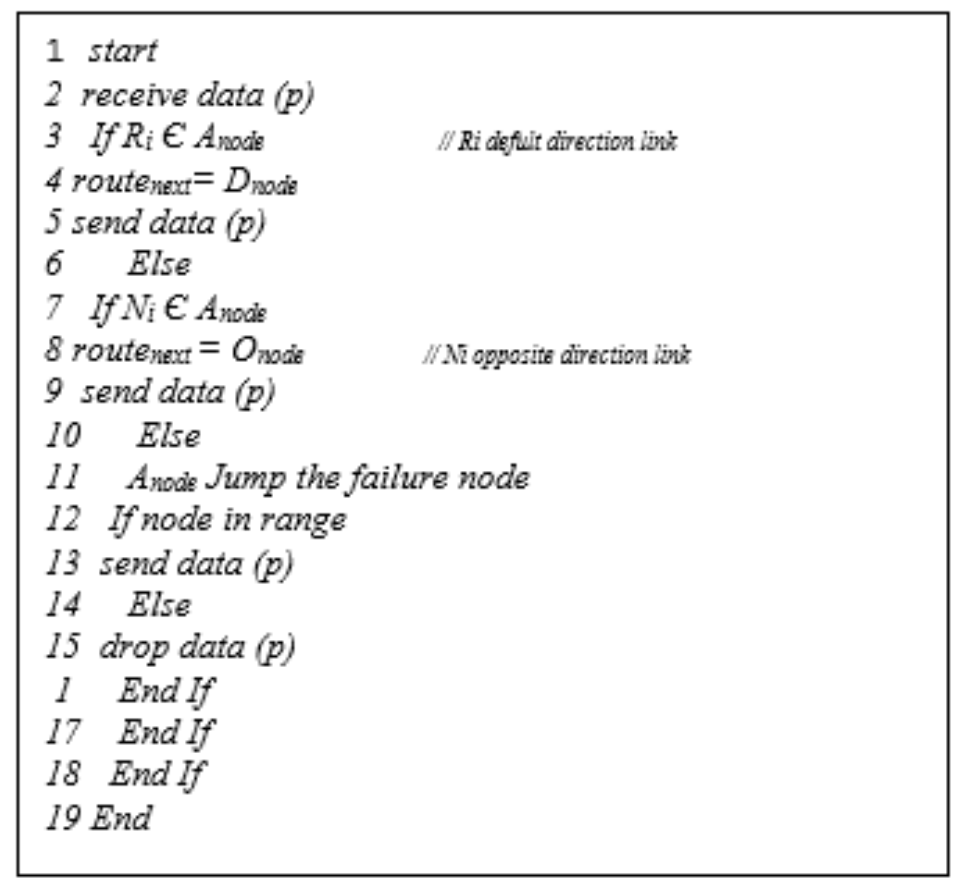

Figure 6. Improved SRJ algorithm for ARNs node 


\section{SIMULATION SCENARIOS AND RESULT}

The proposed work is implemented with the ISRJ algorithm. The main protocol layers of sensor nodes in the network simulation are physical, link, network, and application layers $[15,16]$. In this work, two software tools are used OMNeT++ [17, 18], and Matlab [19] based on their integration. OMNeT++ is a modular and framework simulator for a separate event network supporting C, C ++ language [20, 21]. The application layer, network layer, and link layers are simulated using OMNeT++. The physical layer is simulated using MATLAB functions [22-24]. The modulator in the physical layer is used to generate a binary phase-shift keyed (PSK) waveform. PSK modulation is applied to change the phase of a carrier wave. Table 1 represent the simulation parameters that were adopted in this work. The signal-to-noise-ratio (SNR) for an underwater acoustic signal is modeled in the (1) [25]:

$$
S N R=S L-T L-N L
$$

where $S L$ is the Source Level, $T L$ represents the transmission loss due to the underwater environment which describes the impaired sound over a distance, consists of attenuation and propagation losses, and NL is the Noise Level. The transmission loss can be obtained based on the (2) [25]:

$$
T L=10 \log d+A C 10^{3}+T A
$$

$T A$ is the transmission anomaly, $A C$ is the absorption coefficient, and represents the distance between two nodes (sender, receiver). Based on (1), the value of ambient noise of $10 \mathrm{~dB}$ and an assumed SNR of $15 \mathrm{~dB}$ was assumed at the receiver. Depending on the above assumptions, the source level intensity can be expressed as shown in the (3):

$$
S L=T L+25
$$

Table 1. The parameters of the simulation

\begin{tabular}{cc}
\hline Parameters & Value \\
\hline The length of Pipeline & $1000 \mathrm{~m}$ \\
Type of nodes & BSN,ARN,DRN,DDN \\
Total Number of BSN per ARN & 4 (one node every $50 \mathrm{~m})$ \\
Total Number of DRN & 10 (one node every $100 \mathrm{~m}$ ) \\
Total Number of ARN & 5 (one node every $150 \mathrm{~m})$ \\
Total Number of DDN & 2 (one node every $1000 \mathrm{~m})$ \\
Ranges of nodes & $75.100 .150 \mathrm{~m}$ \\
Bit rate & $10 \mathrm{KHz}$ \\
Packet size & 100 byte \\
Hello Packet & 12 byte \\
Propagation model & Underwater propagation \\
Number of Sinks & 2 \\
simulation time & $1000 \mathrm{~s}$ \\
\hline
\end{tabular}

The end-to-end delay (EED) can be obtained [26]:

$$
E E D=N(\operatorname{tprop}(i, i+1)+t p k t)
$$

where $N$ represent the hops to reach the distnation, $(\operatorname{tprop}(i, i+1))$ is the time of propagation between nodes $(i, \mathrm{i}+1)$ in the underwater environment, and tpkt is the time of data packet transmission. The results are compared between the two strategies (SRJ and ISRJ). The experimental results are compared relying on the average overhead, communication, energy consumption, and end-to-end delay.

\subsection{Average overhead communication}

The average number of control packets sent in the relay nodes is measured as shown in Figure 7. The overhead communication for the ISRJ strategy is approximately 54\% while the overhead communication for the SRJ strategy is higher at approximately $63 \%$. This is because if there is a fault case in relay nodes, the sensor nodes will continue trying to contact other relay nodes and this will cause more routing overhead in the case of SRJ. For ISRJ, the failure of DRN does not affect the sensor nodes as they are connected to the ARNs. 


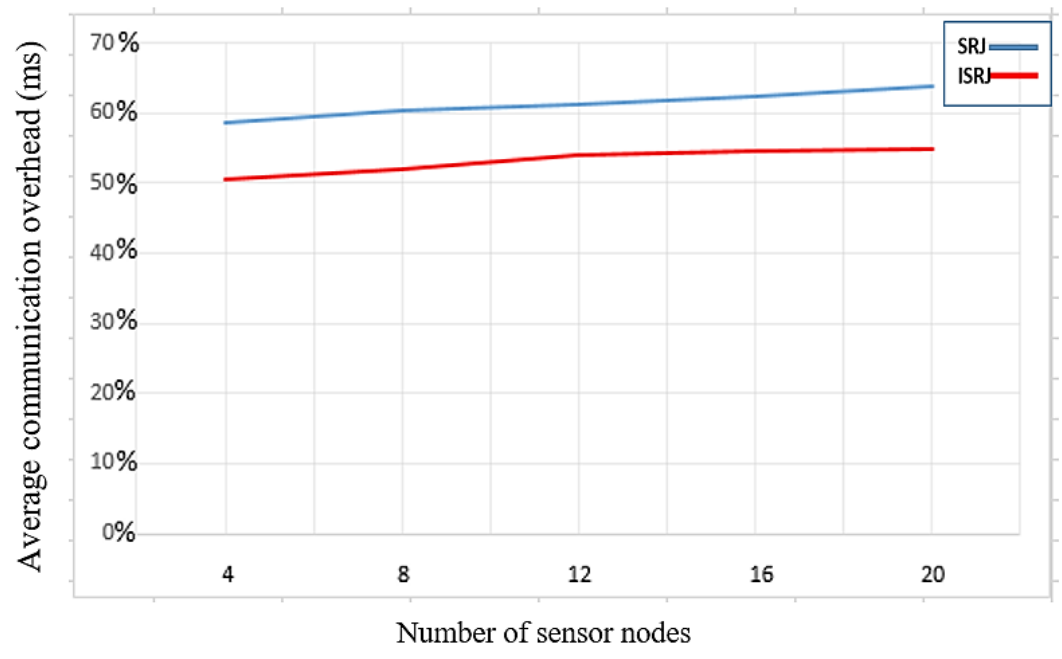

Figure 7. Average overhead communication

\subsection{Energy consumption and end-to-end delay}

Energy efficiency and reliability should be considered when designing and developing UWSN. Reliability requires a high percentage of packet delivery and high productivity so energy consumption must be reduced without sacrificing network productivity. Figure 8 shows the average energy consumption in relay nodes during the total simulation time for each strategy. The results show better performance when using the ISRJ algorithm compared to the SRJ algorithm. For example, in the case of DRN10, the energy consumption is about $632.3 \mu \mathrm{J}$ in the ISRJ strategy while in the SRJ strategy the energy consumed is $813.2 \mu \mathrm{J}$.

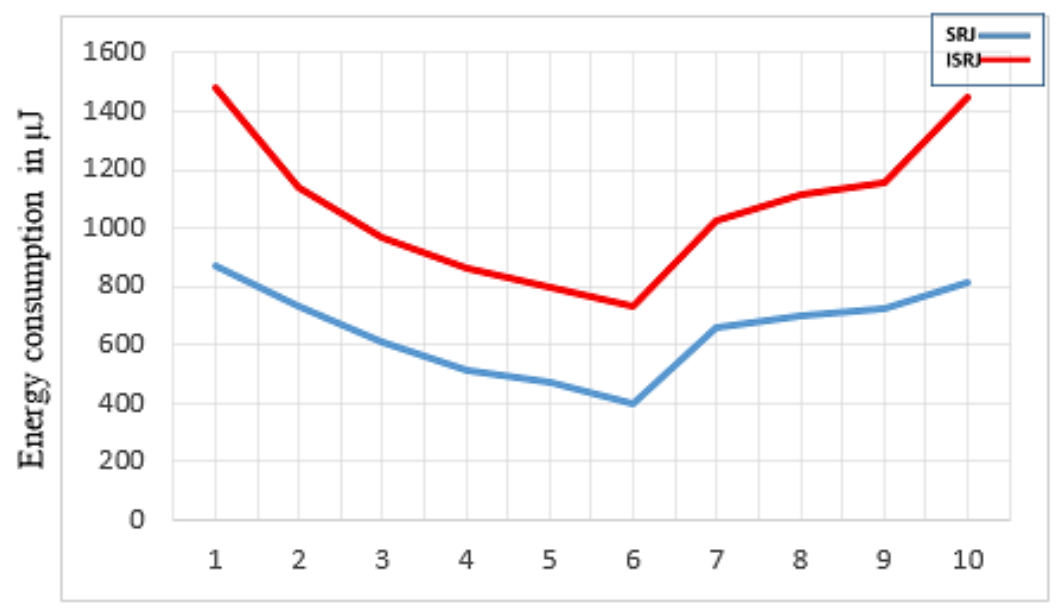

DRN number

Figure 8. Energy consumption in DRN's in both strategies

The graph in Figure 9 represents the energy consumed of each ARN at the total simulation time of an ISRJ scenario. Note that the level of energy consumption in the battery is lower than the energy consumed in the battery of data relay nodes. For instance, the maximum energy consumption of ARNs in this scenario is in node 2 , which is $232.62 \mu \mathrm{J}$, while the lowest energy consumption in node 1 which is $254.3 \mu \mathrm{J}$. This provides an advantage to ISRJ Strategy because the process of working sensor nodes will be longer than the SRJ Strategy. By adding ARN to the hierarchical structure of the new network model, a trade-off should be made between the energy consumed in DRNs and the end-to-end delay depending on the number of hops (how the nodes are deployed over oil pipeline) and the ISRJ algorithm to provide reliability depending on the end-to-end delay and the energy consumption. The end-to-end delay average has been recorded depending on 
the how many active relay nodes is available. For example, in case there are 7 or 8 active relay nodes the average end-to-end delay for SRJ strategy is $1.002 \mathrm{~s}$ and $1.23 \mathrm{~s}$ respectively while in ISRJ strategy is $0.944 \mathrm{~s}$ and $1.2801 \mathrm{~s}$ respectively. Figure 10 represents the end-to-end delay of two strategies.

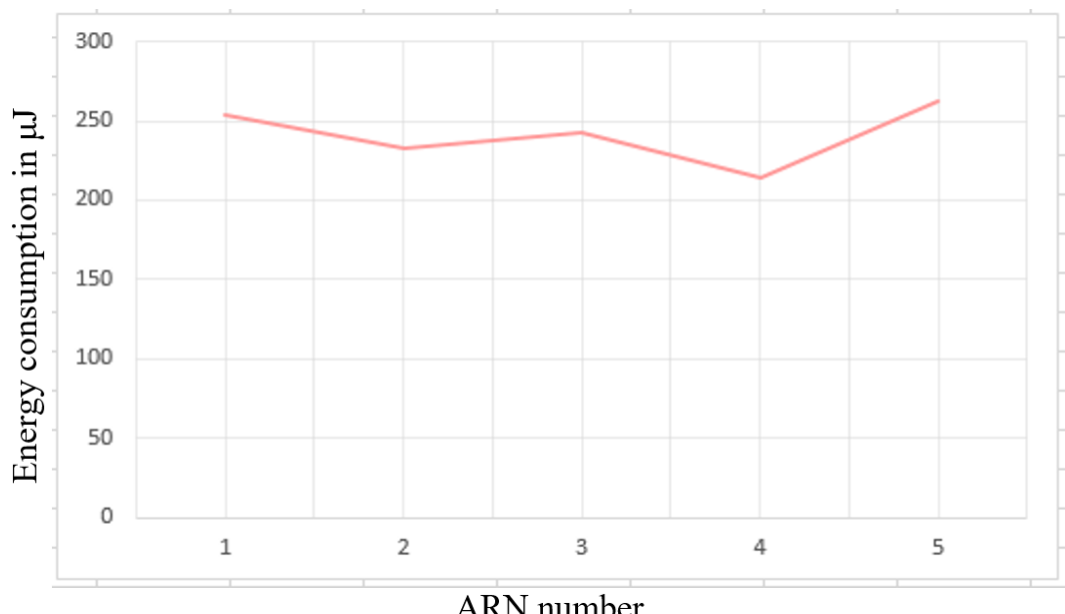

Figure 9. Energy consumption in ARN's in ISRJ strategy

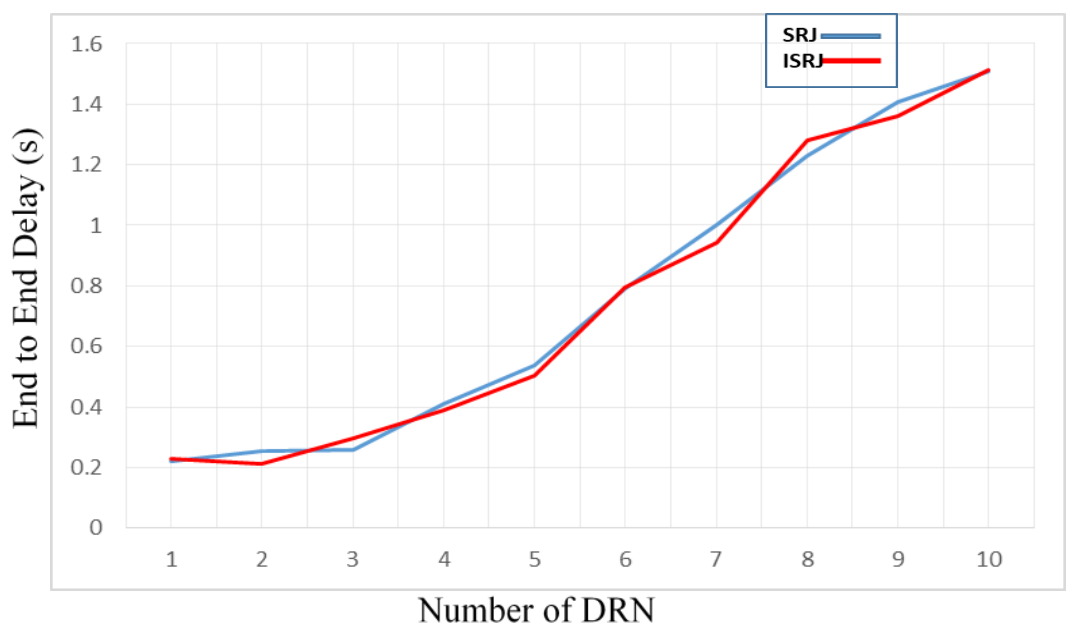

Figure 10. Average end-to-end delay

\section{CONCLUSION}

In this work, the oil pipeline monitoring deployment scheme is designed and implemented with a hierarchical structure of the parent/child system by adding ARN node based on a linear deployment algorithm to deploy nodes that provide complete coverage of the oil pipeline despite the increase in cost. In SRJ strategy the sensor nodes are connected to the relay nodes in the SRJ strategy and if there is a fault case in DRNs, the sensor nodes try to connect other DRN. This increases the control packets, therefore, increases the average overhead communication. The SRJ algorithm based on the number of hops to send packets toward the least energy path is improved and implemented in ARN node by relying on active relay nodes in each directions rather than the number of hops on each side to reduce time and energy consumption and reduce the number of times increased transmission range in DRNs to achieve higher reliability, therefore, the network is fault-tolerant. Where it is noticed that the difference in end-to-end delay between two strategies is in milliseconds in the different cases depending on the number of relay nodes. Thus it is acceptable in a harsh underwater environment. As the average energy consumption decreased by $20.3 \%$ in the network. Trade-offs between energy consumption and end-to-end delay has shown that the network is not much affected by the end-to-end delay, in addition, the energy consumed in the ISRJ strategy is lower than in SRJ strategy. 


\section{ACKNOWLEDGEMENTS}

The authors unanimously thank the reviewers and the journal's staff for their useful instructions that enhance the paper's quality.

\section{REFERENCES}

[1] N. Mohamed, I. Jawhar, J. Al-Jaroodi and L. Zhang, "Monitoring Underwater Pipelines Using Sensor Networks," in 2010 IEEE 12th International Conference on High-Performance Computing and Communications (HPCC), Melbourne, VIC, 2010, pp. 346-353.

[2] C. M. G. Gussen, et al., "A Survey of Underwater Wireless Communication Technologies," JCIS, vol. 31, no. 1, pp. 242-255, 2016.

[3] T. C. Yang, "Properties of underwater acoustic communication channels in shallow water," The Journal of the Acoustical Society of America, vol. 131, no. 1, pp. 129-145, 2012.

[4] P. A. van Walree, et al., "Stretched-exponential Doppler spectra in underwater acoustic communication channels," The Journal of the Acoustical Society of America, vol. 128, no. 5, pp. EL329-EL334, 2010.

[5] S. Varshney, et al., "A comparative study of hierarchical routing protocols in wireless sensor networks," 2015 2nd International Conference on Computing for Sustainable Global Development (INDIACom), New Delhi, 2015, pp. 1018-1023.

[6] G. Tuna and R. Das, "Trade-off Analysis of Underwater Acoustic Sensor Networks," IOP Conference Series: Materials Science and Engineering, vol. 235, 2017, pp. 1-6.

[7] N. Mohamed, et al., "Sensor Network Architectures for Monitoring Underwater Pipelines," Sensors, vol. 11, no. 11 , pp. 10738-10764, 2011

[8] M. Z. Abbas, et al., "Scalable Nodes Deployment Algorithm for the Monitoring of Underwater Pipeline," TELKOMNIKA Telecommunication Computing Electronics and Control, vol. 14, no. 3, pp. 1183-1192, 2016.

[9] Z. Ahmed and K. A. Bakar, "An Enhanced Underwater Linear Wireless Sensor Network Deployment Strategy for Data Collection," International Journal of Innovative Computing, vol. 8, no. 4, pp. 39-45, 2018.

[10] E. F. Farid, A. A. Mahmoud and A. M. Bahaa-Eldin, "Adaptive Smart Redirect and Jump Algorithm (ASRJ) for Wireless Sensor Networks," in 2015 Tenth International Conference on Computer Engineering \& Systems (ICCES), Cairo, Egypt, 2015, pp. 247-252.

[11] N. Mohamed, I. Jawhar, J. Al-Jaroodi and L. Zhang, "Monitoring Underwater Pipelines Using Sensor Networks," in 2010 IEEE 12th International Conference on High-Performance Computing and Communications (HPCC), Melbourne, VIC, 2010, pp. 346-353.

[12] M. Zimmerling, "Energy-efficient routing in linear wireless sensor networks," in Mobile Adhoc and Sensor Systems, 2007.

[13] Z. Guo, et al., "GRE: Graded Residual Energy Based Lifetime Prolonging Algorithm for Pipeline Monitoring Sensor," in 2008 Ninth International Conference on Parallel and Distributed Computing, Applications and Technologies, 2008, pp. 203-210.

[14] I. Jawhar, et al., "Data Communication in Linear Wireless Sensor Networks Using Unmanned Aerial Vehicles," 2014 International Conference on Unmanned Aircraft Systems (ICUAS), Orlando, 2014, pp. 43-51.

[15] M. Zimmerling, et al., "Localized power-aware routing in linear wireless sensor networks," in CASEMANS, 2008.

[16] T. Rault, et al., "Energy efficiency in wireless sensor networks: A top-down survey," Computer Networks, vol. 67, pp. 104-122, 2014.

[17] “The OMNeT++/MiXiM framework-User Manual," 2015. [Online]. Available: http://mixim. sourceforge.net/.

[18] Proakis J. G., "Digital communications," 3rd ed., New York, McGraw Hill, 1995.

[19] M. Murad, et al., "A Survey on Current Underwater Acoustic Sensor Network Applications," IJCTE, vol. 7, no. 1, pp. 51-56, 2014.

[20] B. Hasannezhad, "Simulation of Mobile Hydroacoustic Communications in Underwater Acoustic Sensor Networks," Master of Computer Science, Carleton University, Ottawa, Ontario, 2015.

[21] Abdelkareem A. E., et al., "Adaptive time varying Doppler shift compensation algorithm for OFDM-based underwater acoustic communication systems," Ad Hoc Networks, vol. 45, pp. 104-119, 2016.

[22] I. MathWorks, "MATLAB: the language of technical computing," Desktop tools and development environment, version 7, vol. 9, 2005.

[23] Abdelkareem A. E., et al., "Design and Implementation of an Embedded System for Software Defined Radio," International Journal of Electrical and Computer Engineering (IJECE), vol. 7, no. 6, pp. 3484-3491, 2017.

[24] B. S. Borowski, "Application of channel estimation to underwater," Acoustic communication PhD thesis, Stevens Institute of Technology, Castle Point on Hudson, Hoboken NJ, 2010.

[25] M. Barbeau, et al., "Simulation of Underwater Communications with Colored Noise Approximation and Mobility," 2015 IEEE 28th Canadian Conference on Electrical and Computer Engineering (CCECE), Halifax, 2015, pp. $1532-1537$.

[26] W. A. Kuperman and P. Roux, "Underwater Acoustics," in T. D. Rossing (ed), Springer Handbook of Acoustics, New York, Springer New York, 2014. 


\section{BIOGRAPHIES OF AUTHORS}
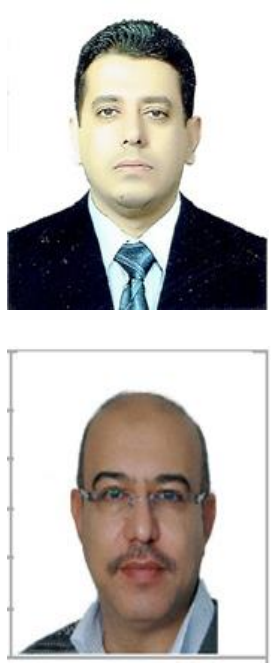

Waseem M. Jassim Alshaheen is graduated of Computer Engineering from the University of Basrah Iraq (2003). Received his master's degree in the network engineering and international internet technology from University of Alnahrain Baghdad (2020). His interest fields are in the Information and Communication Technology. Curently, he is working in Basrah oil company (BOC).

E-mail: wassim.mohammed@ coie-nahrain.edu.iq,wassim.hwm@gmail.com.

Ammar E. Abdelkareem Al-Qassab received a degree in computer engineering in 1992 from University of Technology, Baghdad and a M.Sc. computer engineering in 2003 from University of Technology, Baghdad. He received a Ph.D. degree in computer engineering from Newcastle University,UK in 2012. His teaching experience in computer architecture and microprocessors|, computer networks, mobile wireless communications, networks embedded systems, modern networks technologies, digital signal processing and wireless communications. Currently, he is Asst. Professor at network engineering department, College of Information Engineering, Alnahrain universty.

E-mail: : ammar.e@coie-nahrain.edu.iq,ammar.algassab@gmail.com. 